\title{
Investigation of anti-cancer mechanisms by comparative analysis of naked mole rat and rat
}

\author{
Zhiyuan Yang ${ }^{1}$, Yan Zhang ${ }^{2 *}$, Luonan Chen ${ }^{1,3^{*}}$ \\ From The 6th International Conference on Computational Systems Biology (ISB2012) \\ Xi'an, China. 18-20 August 2012
}

\begin{abstract}
Background: The naked mole rats (NMRs) are small-sized underground rodents with plenty of unusual traits. Their life expectancy can be up to thirty years, more than seven times longer than laboratory rat. Furthermore, they are resistant to both congenital and experimentally induced cancer genesis. These peculiar physiological and pathological characteristics allow them to become a suitable model for cancer and aging research.

Results: In this paper, we carried out a genome-wide comparative analysis of rat and NMR using the recently published genome sequence of NMR. First, we identified all the rat-NMR orthologous genes and specific genes within each of them. The expanded and contracted numbers of protein families in NMR were also analyzed when compared to rat. Seven cancer-related protein families appeared to be significantly expanded, whereas several receptor families were found to be contracted in NMR. We then chose those rat genes that were inexistent in NMR and adopted KEGG pathway database to investigate the metabolic processes in which their proteins may be involved. These genes were significantly enriched in two rat cancer pathways, "Pathway in cancer" and "Bladder cancer". In the rat "Pathway in cancer", 9 out of 14 paths leading to evading apoptosis appeared to be affected in NMR. In addition, a significant number of other NMR-missing genes enriched in several cancer-related pathways have been known to be related to a variety of cancers, implying that many of them may be also related to tumorigenesis in mammals. Finally, investigation of sequence variations among orthologous proteins between rat and NMR revealed that significant fragment insertions/deletions within important functional domains were present in some NMR proteins, which might lead to expressional and/or functional changes of these genes in different species.
\end{abstract}

Conclusions: Overall, this study provides insights into understanding the possible anti-cancer mechanisms of NMR as well as searching for new cancer-related candidate genes.

\section{Background}

The naked mole rats (NMRs, Heterocephalus glaber) are mouse-sized subterranean rodents native to East Africa [1]. They have an exceptional set of physiological traits that make them adapt to living in the underground of

\footnotetext{
* Correspondence: yanzhang01@sibs.ac.cn; Inchen@sibs.ac.cn

${ }^{1}$ Key Laboratory of Systems Biology, SIBS-Novo Nordisk Translational

Research Centre for PreDiabetes, Shanghai Institutes for Biological Sciences, Chinese Academy of Sciences, Shanghai 200031, China

${ }^{2}$ Key Laboratory of Nutrition and Metabolism, Institute for Nutritional Sciences, Shanghai Institutes for Biological Sciences, Chinese Academy of Sciences, Shanghai 200031, China

Full list of author information is available at the end of the article
}

droughty desert. They are becoming one of the most extraordinary organisms known to science [2].

NMRs are the longest-lived rodent known till now and their maximum lifespan can be up to thirty years [3]. By contrast, other similar-sized rodents such as mouse possess a life expectancy of only four years, which is far less than that of NMR. Previously published studies have indicated that the longevity of NMR was possibly because of the negligible decrement of age-related physiological characteristic along with their lifetime, such as declining fertility and mortality rate [4].

Besides delayed senescence, NMRs are remarkably resistant to both congenital and experimentally induced
C Biomed Central

C 2013 Yang et al.; licensee BioMed Central Ltd. This is an open access article distributed under the terms of the Creative Commons Attribution License (http://creativecommons.org/licenses/by/2.0), which permits unrestricted use, distribution, and reproduction in any medium, provided the original work is properly cited. 
cancer genesis [5]. Cancer is a group of complex polygenic diseases that commonly affect lots of vertebrates, and is constantly considered to be an inevitable accompanied by senescence. Cancer is the second dominant cause of mortality in the world, which cause 7.6 million of death estimated by World Health Organization [6]. It has been recognized for quite a long time that cancer genesis is closely related to tumour suppressor genes and oncogenes [7]. Identifying the function of the added genes may bring us in another way to explore the regulatory network of the cancer process. In addition, the mechanisms of cancer resistance present in NMR are not thoroughly clear. Thus, identification of NMR genes closely implicated in cancer may provide us effective clues for delineating causes of cancer proneness and studying anti-cancer properties for mammalian organisms.

NMRs possess several other special physiological characteristics as well. Although NMRs belong to the order of rodentia, they are actually poikilothermal animals whose body temperatures vary continuously following the environment [8]. Furthermore, NMRs are insensitive to certain types of pain [9] and acid [10], and are well adapted to the underground surrounding at an extremely low oxygen concentration (10\%-15\%) [11].

Recently, using high-throughput next generation sequencing techniques, the genome of NMR has been sequenced. These excellent resources provide great opportunities for understanding the exceptional characteristics of NMR and improve biological and biomedical studies. Previously, some genes have been identified to be related to some of these unusual characteristics, e.g., the telomerase reverse transcriptase (TERT) gene and some other genes, which may be involved in extended longevity mechanisms of NMR [12]. However, investigation of the genomic information of NMR at a systems-biology level is still lacking, which may provide additional information to uncover the molecular mechanisms for the extraordinary traits (e.g., anti-cancer) of NMR.

In this paper, a comparative genomics study was carried out to explore the genes that were either common between rat and NMR, or specific to each of them. We divided these genes into three groups: common genes, genes only present in NMR and genes only present in rat. We then used the Pfam database to identify the events of gain or loss of different protein families between these two species. In addition, the Kyoto Encyclopedia of Genes and Genomes (KEGG) database was used to study the rat pathways in which the NMR-missing genes participate. A significant number of these genes were found to be enriched in the pathways related to the exceptional characteristics of NMR (such as cancer pathways), many of which have been previously reported to be associated with various cancers. Finally, we analyzed the sequence variations (such as domain insertion/deletion) of orthologous proteins to investigate the potentially expressional and/or functional alternations of them between rat and NMR. Overall, our data not only help unveil the cancer resistance mechanisms of NMR but provide insights into identifying new cancer-related genes.

\section{Methods}

\section{Genome, database and resources}

The complete set of annotated rat and NMR protein sequences were obtained from the UniProt database (http://www.uniprot.org/). For those genes with alternative splicing variants, proteins with the smallest PE value (which means the most possibility for the existence of the proteins) and the longest length were chosen to represent the gene-encoding protein sequences. A total of 20835 and 21553 proteins corresponding to their genes were finally obtained for rat and NMR, respectively.

The file containing the whole pathways of rat was downloaded from the KEGG database (http://www. genome.jp/kegg/pathway.html). The Online Mendelian Inheritance in Man (OMIM) database (http://www. ncbi.nlm.nih.gov/omim) was used to analyze the relationship between cancers and human orthologs of genes absent in NMR. Furthermore, the expression data of these genes in rat tissues were obtained from the Gene Expression Atlas (GXA) database (http://www.ebi.ac.uk/gxa/), which was used to identify whether or not the expression levels of these genes were related to cancer development.

\section{Identification of orthologous genes between rat and NMR}

To analyze the orthologous gene pairs between rat and NMR, we employed the complete set of annotated proteins of one organism as queries to search for orthologs in the other species via BLASTP with a cut-off of E-value $\leq 1 \mathrm{e}-6$. Orthologous genes were further defined as bidirectional best hits.

On the basis of identified orthologs between rat and NMR, we dissected all the genes into three classifications:

(1) Class I: the Shared genes, which were shared between rat and NMR;

(2) Class II: the NMR-missing genes, which were absent in NMR but present in rat;

(3) Class III: the NMR unique genes, which were found in NMR but missing in rat.

\section{Pfam analysis}

Considering that the conserved domains in a protein could provide information for its function and evolutionary dynamics, we used the Pfam database [13] (http:// pfam.sanger.ac.uk/search), which collected a large 
collection of protein families, to search for gain or loss events of different protein families between these two species.

All the proteins of rat and NMR were searched against Pfam database with a cut-off of E-value $\leq 1 \mathrm{e}-5$. For each protein, if two or more Pfam families were available, only the one with the smallest E-value was selected. The number of each protein family in rat and NMR was then calculated respectively.

\section{KEGG pathway analysis of Class II genes}

We further dissected the pathways containing Class II genes using the KEGG database resource, which is a collection of manually curated pathway maps according to current knowledge on protein-protein interactions [14].

First, each gene of Class II was mapped to their pathways. The p-value of the enrichment of NMR-missing genes in each pathway was then calculated by hypergeometric distribution test. Moreover, considering that KEGG pathways were composed of nodes which were actually modules including single gene or multiple functionally similar genes, we further analyzed the number and percentage of the nodes containing Class II genes in each of the enriched pathways.

\section{Sequence variation analysis of Class I genes}

Although Class I genes were considered as orthologous genes between rat and NMR, sequence variations had been previously observed for certain proteins. For example, the glutathione peroxidase 1 (GPx1), which is highly expressed in mouse liver and kidney, has an early stop codon in NMR. Such a variation results in the lack of the C-terminal part and may be related to an order of magnitude lower activity in NMR tissues [15]. Thus, it would be useful to further study the orthologous genes between the two organisms for potential changes with regard to their function and/or regulation.

To systematically investigate such deletion or insertion events, we analyzed the BLASTP sequence alignment results for each Class I gene in NMR, and focused on gap-related parameters, such as alignment length, number of mismatches, and percentage of identical matches, to calculate the lengths of sequence insertions/deletions. Protein pairs of rat and NMR proteins were chosen at a cut-off of gap length $>25$ and percentage of mismatches $<10 \%$. To avoid incorrect protein annotation of NMR, the NMR proteins with significant fragment deletion were searched against the NMR genome using TBLASTN for further verification of the absence of these segments. Finally, all selected proteins were searched against the Conserved Domains Database (http://www.ncbi.nlm.nih.gov/Structure/cdd/cdd.shtml) to identify their functional domains.

\section{Results}

\section{Distribution of orthologous genes in rat and NMR}

Figure 1 shows the Venn diagram between rat and NMR genes. A set of 15408 genes (Class I) were found in both rat and NMR, which occupied $73.9 \%$ of the total predicted NMR genes. On the other hand, 6145 rat genes (Class II) lacked orthologs in NMR. In contrast, 5427 genes (Class III) appeared to be unique in NMR. Nevertheless, considering that the number of NMR orthologous genes (Class I) is only about $71.5 \%$ of that of the rat total genes, it is possible that some genes may be mis-annotated in the currently released version of NMR genome.

\section{Pfam analysis of rat and NMR genes}

All the annotated proteins of rat and NMR were searched against Pfam database (containing 13672 families) for the classification into different protein families. 2442 and 2523 protein families (with 2416 overlapped families) were obtained in rat and NMR, respectively, indicating that the two species shared almost the same protein families.

We further analyzed the gain or loss events for each protein family in NMR. Table 1 shows the top 15 families sorted by the number of expanded genes (gain) in NMR. Among them, two distinct cancer-related protein families: "Melanoma-associated antigen family" and "Mortality factor 4 families" had significant gene expansion in NMR. A total of 56 and 15 genes were found for the two families respectively in NMR, which were 27 and 11 more than those in rat. Besides, several other families shown in Table 1 have also been reported to be related to cancer, such as the protein kinase $\mathrm{C}$ (PKC) family and several heat shock proteins (HSP70 and HSP90).

The PKC family, which possessed 53 proteins in NMR, had variable roles in tumour biology depending on the intracellular localizations and cell types. PKCs were generally abnormally regulated in the cancers of the breast, prostate, kidney and liver [16], and remained

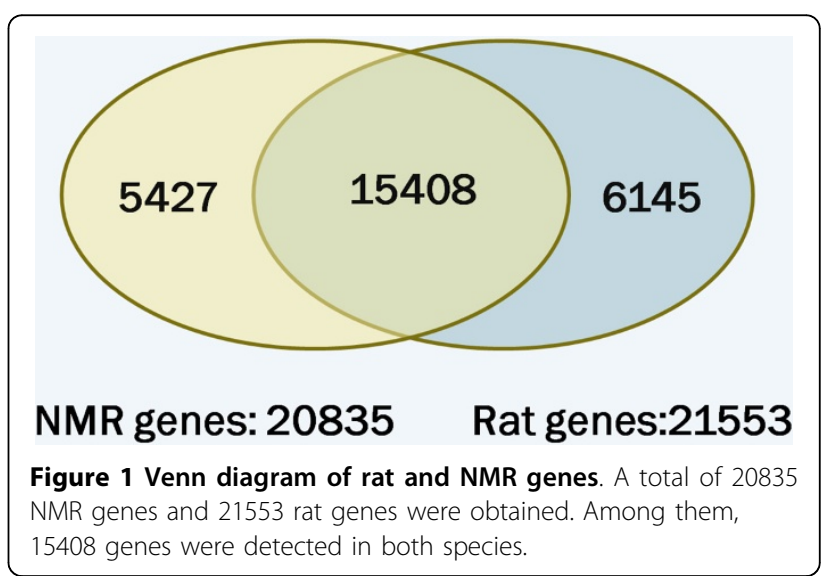


Table 1 Expanded Pfam family of NMR

\begin{tabular}{|c|c|c|c|c|c|}
\hline PFAM entry & Explanation & class I genes & class II genes & class III genes & expanded \\
\hline PF00001 & rhodopsin family & 221 & 108 & 205 & 97 \\
\hline PF01454 & Melanoma-associated antigen family & 11 & 18 & 45 & 27 \\
\hline PF00433 & Protein kinase C family & 26 & 1 & 27 & 26 \\
\hline PF02093 & Gag P30 core shell protein & 1 & 1 & 27 & 26 \\
\hline PF00012 & Hsp70 protein & 13 & 0 & 23 & 23 \\
\hline PF01849 & NAC domain & 4 & 2 & 20 & 18 \\
\hline PF00276 & Ribosomal protein L23 & 1 & 1 & 16 & 15 \\
\hline PF01282 & Ribosomal protein S24e & 2 & 2 & 16 & 14 \\
\hline PF05712 & Mortality factor 4 family & 2 & 2 & 13 & 11 \\
\hline PF00261 & Tropomyosin & 5 & 0 & 10 & 10 \\
\hline PF00153 & Mitochondrial carrier protein & 46 & 8 & 17 & 9 \\
\hline PF00118 & TCP-1/cpn60 chaperonin family & 12 & 2 & 10 & 8 \\
\hline PF00183 & Hsp90 protein & 4 & 0 & 8 & 8 \\
\hline PF01015 & Ribosomal S3Ae family & 1 & 1 & 9 & 8 \\
\hline PF01873 & Domain found in IF2B/IF5 & 2 & 0 & 8 & 8 \\
\hline
\end{tabular}

The Pfam families were sorted by the expanded number of protein families. In this table, we only showed the top 15 families.

Table 2 Contracted Pfam family of NMR

\begin{tabular}{|c|c|c|c|c|c|}
\hline PFAM entry & Explanation & class I genes & class II genes & class III genes & contracted \\
\hline PF13853 & Olfactory receptor & 293 & 852 & 691 & 161 \\
\hline PF03402 & Vomeronasal organ receptor & 2 & 99 & 3 & 96 \\
\hline PF00003 & class C G-protein-coupled receptors & 14 & 72 & 17 & 55 \\
\hline PF00028 & Cadherin family & 50 & 38 & 4 & 34 \\
\hline PF05296 & Mammalian taste receptor protein & 4 & 32 & 2 & 30 \\
\hline PF08391 & Ly49-like protein & 1 & 21 & 0 & 21 \\
\hline PF02994 & L1 transposable element & 0 & 20 & 0 & 20 \\
\hline PF01157 & Ribosomal protein L21e & 1 & 32 & 13 & 19 \\
\hline PF13885 & high sulfur B2 protein & 3 & 12 & 1 & 11 \\
\hline PF13841 & Beta defensin & 7 & 11 & 0 & 11 \\
\hline PF01198 & Ribosomal protein L31e & 1 & 16 & 6 & 10 \\
\hline PF01779 & Ribosomal L29e protein family & 1 & 10 & 1 & 9 \\
\hline PF12774 & chaperone-like ATPases & 1 & 9 & 0 & 9 \\
\hline PF00879 & Defensin propeptide & 0 & 9 & 1 & 8 \\
\hline PF00618 & Ras-like small GTPase & 0 & 8 & 0 & 8 \\
\hline
\end{tabular}

The Pfam families were sorted by the contracted number of protein families. In this table, we only showed the top 15 families.

as a possible target for cancer prevention and therapy [17]. Here, a total of 27 additional PKC members were identified in NMR, implying that these new PKCs may play an important role in preventing NMR from cancer.

HSP proteins are a group of functionally related proteins regulating protein folding and unfolding reactions. HSP70 proteins were reported to be overexpressed in the malignant melanoma [18]. On the other hand, HSP90 proteins were also implicated to be involved in breast cancer progression because of its overexpression in breast cancer cell lines and association with survival of breast cancer [19]. Thus, HSP70 and HSP90 proteins have been considered as the useful targets for cancer therapy [20,21]. In this study, the significant expansions of members of these two families in NMR were consistent with their potential roles in cancer prevention and may provide clues for the anti-cancer trait of NMR.

Table 2 shows the top 15 protein families with contracted gene numbers (loss) in NMR. Among them, several protein families have been previously verified to be involved in cancer development. For example, the cadherin family is a class of type-1 trans-membrane proteins. Members of this family may play important roles in cellcell adhesion in different organ systems [22]. All kinds of abnormal expression of cadherin family proteins had been reported as a widespread phenomenon in mammary cancer and these proteins had been frequently implicated in tumour progression [23]. The loss of so many genes 
in this family may also contribute to the complex cancerresistant mechanisms of NMR. In addition, a significant loss of members of some receptor protein families, such as the vomeronasal organ receptor (VOR), olfactory receptor and class C G-protein-coupled receptor, may be important for some unique features of the most unusual rodent.

\section{Pathway analysis of NMR-missing genes}

Among 216 rat pathways in the KEGG database, 16 of them were found to be significantly ( $\mathrm{p}$-value $<0.01$ ) enriched by the NMR-missing genes (group II genes) (Table 3). For example, at least 31 rat genes $(10.7 \%)$ in the "Neuroactive ligand-receptor interaction" pathway were not detected in NMR. The loss of these genes might be one of the major reasons why NMR is insensitive to pain and acid [24]. In addition, NMR-missing genes were also significantly enriched in two known cancer pathways, "Bladder cancer" and "Pathways in cancer" (with the p-value 2.37e-3 and 1.17e-3, respectively).

Among the rest of pathways shown in Table 3 five of them were thought to be cancer-related, which include "Cytokine-cytokine receptor interaction" [25], "p53 signalling pathway"[26], "Apoptosis", "Natural killer cell mediated cytotoxicity" [27], "Wnt signalling pathway" [28]" and "Notch signalling pathway" [29]. It is possible that several of these NMR-missing genes are associated with cancer development in other mammals including humans and could be considered as candidate cancerrelated genes.

As KEGG pathways are composed of nodes which may have single or multiple functionally similar genes, we also calculated the percentage of the nodes which contain at least one NMR-missing gene in each of these pathways, and obtained almost the same enriched pathways, such as "Pathways in cancer", "Neuroactive ligandreceptor interaction" and "Oxidative phosphorylation", implying that the pathway enrichment of NMR-missing genes was significant at both gene and node levels (Supplementary Table 1 [see additional file 1]).

Analysis of cancer-related genes that were absent in NMR To investigate the potential mechanisms of the anti-cancer aspects of NMR, three cancer-related pathways, including "pathways in cancer", "MAPK (mitogen-activated protein kinase) signalling pathway" and "Wnt signalling pathway", were chosen as examples for further analysis.

\section{1) Pathways in cancer}

Twenty-nine genes in this pathway were not detected in NMR (Supplementary Table 2 [see additional file 1]). These genes were found to be strongly related to cancer. Half of them correspond to various phenotypes of cancer (e.g., leukemia, lung cancer and adrenal cortical carcinoma) based on the information retrieved from OMIM database, including several well-studied carcinogenesis genes (Bcl2, Casp8, Fas). Moreover, some important proto-oncogenes, such as Myc and Hras1, were also absent. In fact, it is well known that proto-oncogenes are normal genes that could become the oncogenes because of their overexpression or mutations. The loss of the proto-oncogenes in NMR cells may also contribute to cancer resistance.

Among all 29 NMR-missing genes in this pathway, 19 of them $(65.5 \%)$ were previously reported to display differential gene expression levels between cancer and normal tissues. Thus, the absence of these genes might play important roles in suppressing cancer.

Table 3 Pathway enrichment analysis of NMR-missing genes*

\begin{tabular}{|c|c|c|c|c|}
\hline pathway ID & pathway name & gene number & total number & p-value \\
\hline rno04740 & Olfactory transduction & 518 & 1015 & $1.4 \mathrm{E}-11$ \\
\hline rno03010 & Ribosome & 18 & 91 & 4.4E-07 \\
\hline rno04060 & Cytokine-cytokine receptor interaction & 31 & 247 & 2.0E-06 \\
\hline rno04080 & Neuroactive ligand-receptor interaction & 31 & 290 & $5.1 \mathrm{E}-05$ \\
\hline rno00190 & Oxidative phosphorylation & 26 & 244 & $2.1 \mathrm{E}-04$ \\
\hline rno04115 & p53 signaling pathway & 12 & 80 & $5.6 \mathrm{E}-04$ \\
\hline rno05200 & Pathways in cancer & 29 & 317 & $1.2 \mathrm{E}-03$ \\
\hline rno04330 & Notch signaling pathway & 8 & 47 & 2.0E-03 \\
\hline rno05014 & Amyotrophic lateral sclerosis & 10 & 69 & $2.0 \mathrm{E}-03$ \\
\hline rno04610 & Complement and coagulation cascades & 10 & 69 & $2.0 \mathrm{E}-03$ \\
\hline rno05219 & Bladder cancer & 7 & 38 & $2.4 \mathrm{E}-03$ \\
\hline rno04310 & Wnt signaling pathway & 16 & 149 & $3.0 \mathrm{E}-03$ \\
\hline rno04650 & Natural killer cell mediated cytotoxicity & 14 & 125 & $3.6 \mathrm{E}-03$ \\
\hline rno03050 & Proteasome & 8 & 52 & $3.8 \mathrm{E}-03$ \\
\hline rno04210 & Apoptosis & 12 & 107 & $6.8 \mathrm{E}-03$ \\
\hline rno04010 & MAPK signaling pathway & 19 & 213 & $9.9 \mathrm{E}-03$ \\
\hline
\end{tabular}

*: In this table, we only showed the pathways with $p$-value $\leq 0.01$. 
Figure 2 shows the main part of the map of "pathways in cancer". One of the key mechanisms for cancer development is "Evading apoptosis", which is a crucial oncogenic property of cancer cells. Among the 14 paths leading to evasion of apoptosis illustrated in this map, 9 of them appeared to be influenced by direct connection to the nodes which contain NMR-missing genes. Particularly, the nodes of Survivin, Bcl-2, Bcl-XL and Mtor have lost several functionally similar genes in NMR. It is possible that these changes in NMR might block the major routes to evade apoptosis, and then induce the early programmed death of cancer cells as an important cancer barrier.

Recently, a two-tier anti-cancer mechanism associated with contact inhibition regulated by $\mathrm{p} 16^{\text {Ink4a }}$ and $\mathrm{p} 27^{\text {Kip } 1}$ has been reported in NMR [30]. However, rat cells were found to only have contact inhibition regulated by $\mathrm{p} 27^{\mathrm{Kip} 1}$. This is consistent with our results as rats only have $\mathrm{p} 27^{\mathrm{Kip} 1}$ gene. Thus, NMR appeared to have additional unique protective mechanisms for cancer resistance.

\section{2) MAPK signalling pathway}

The MAPK signalling pathway is an important pathway how proteins in the cytoplasm communicate the signals from the receptors on the cell membrane to the nucleus. It is in the central of a molecular metabolic network that mediates cell differentiation and proliferation. In mammalian cells, the MAPK pathway contains three major groups of proteins, including Erk (extracellular

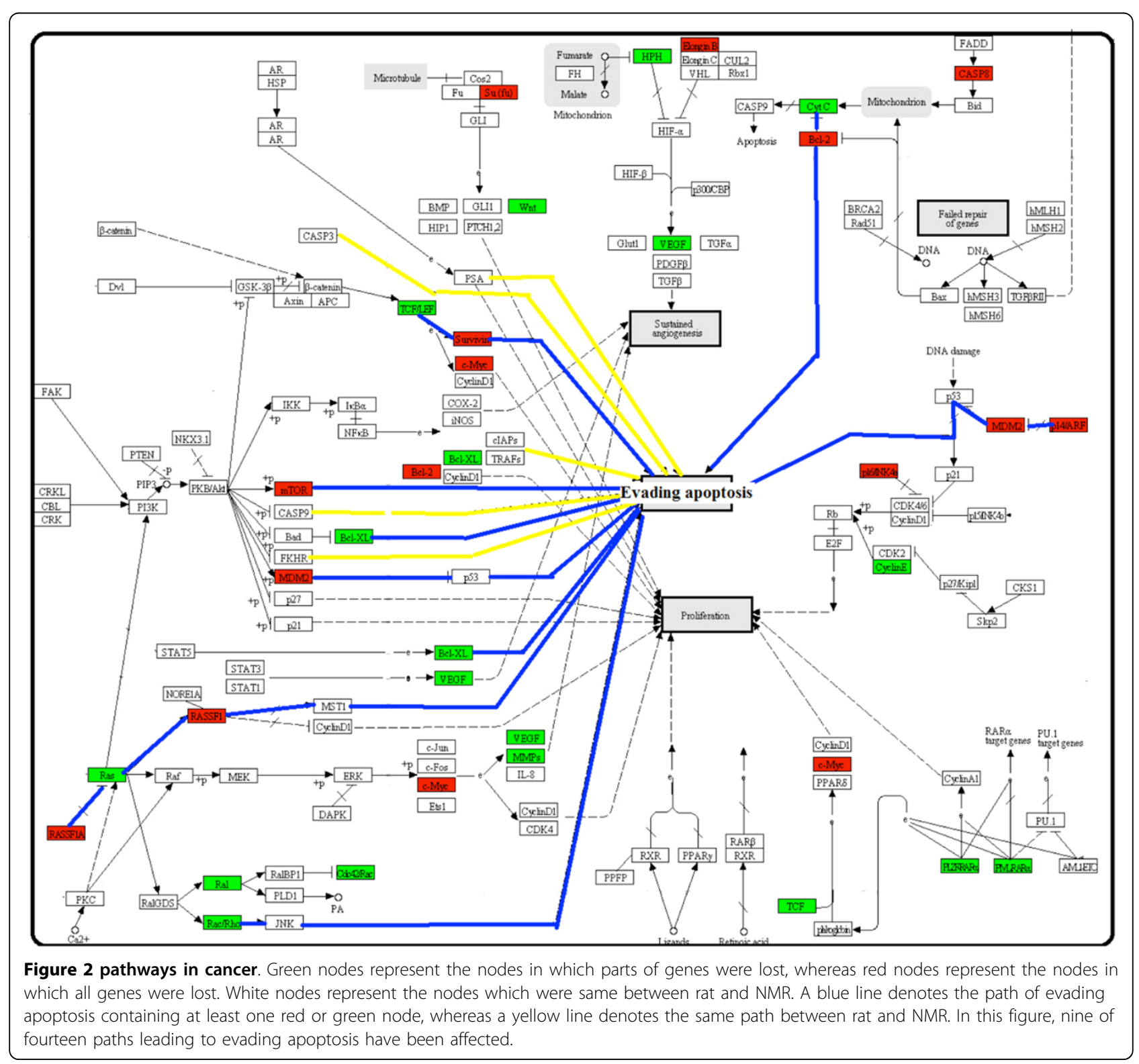


signal-regulated kinase), p38 kinase and Sapk (stress activated protein kinase). These proteins are abnormally regulated in various diseases, including cancer and inflammation.

In this study, 19 genes were found to be absent in NMR (Figure 3 and Supplementary Table 3 [see additional file 1]). By retrieving the OMIM database and GXA database, we found that 7 (36.8\%) of them have been reported to be related to various phenotypes of cancer in other mammals and $10(52.6 \%)$ of them had differential expression levels between cancer and normal tissues.

In this pathway, three proto-oncogenes, Hras1, Myc and Pdgfb (also present in the "Pathways in cancer" pathway), were absent in NMR. It has been previously shown that when one of these genes was mutated, the activity of their enzymes could be stuck in the "on" or "off" position, which was an essential step during the development of many cancers [31]. Recently, it has also been reported that NMR and rat cells acted totally opposed if transfected with Hras1. NMR cell cycle came to an abrupt end as the presence of abnormal chromatin material and anaphase bridges and, while transfected rat cell grew rapidly and formed tumours eventually [32]. Therefore, the loss of these genes might also play a significant role in cancer resistance.

Although the phenotypes of cancer could not be found for other NMR-missing genes, some of them have been demonstrated to be related to the survival of cancer cells, such as Jund and Park genes. Jund is an AP-1 family member involved in various biological processes such as cell apoptosis and tumour metastasis, and could regulate survival of tumour cells in prostate cancer [33]. Prak is a protein kinase, which was previously shown to be implicated in the suppression of skin carcinogenesis [34]. Further experiments are needed to investigate the relationship between these genes and tumorigenesis.

\section{3) Wnt signalling pathway}

The "Wnt signalling pathway" is a conserved proteinprotein interaction network that regulates cell fate decisions and cell-cell communication. This pathway plays a significant role in maintaining stability of internal environment by regulating cell niche in vivo. Abnormal regulation

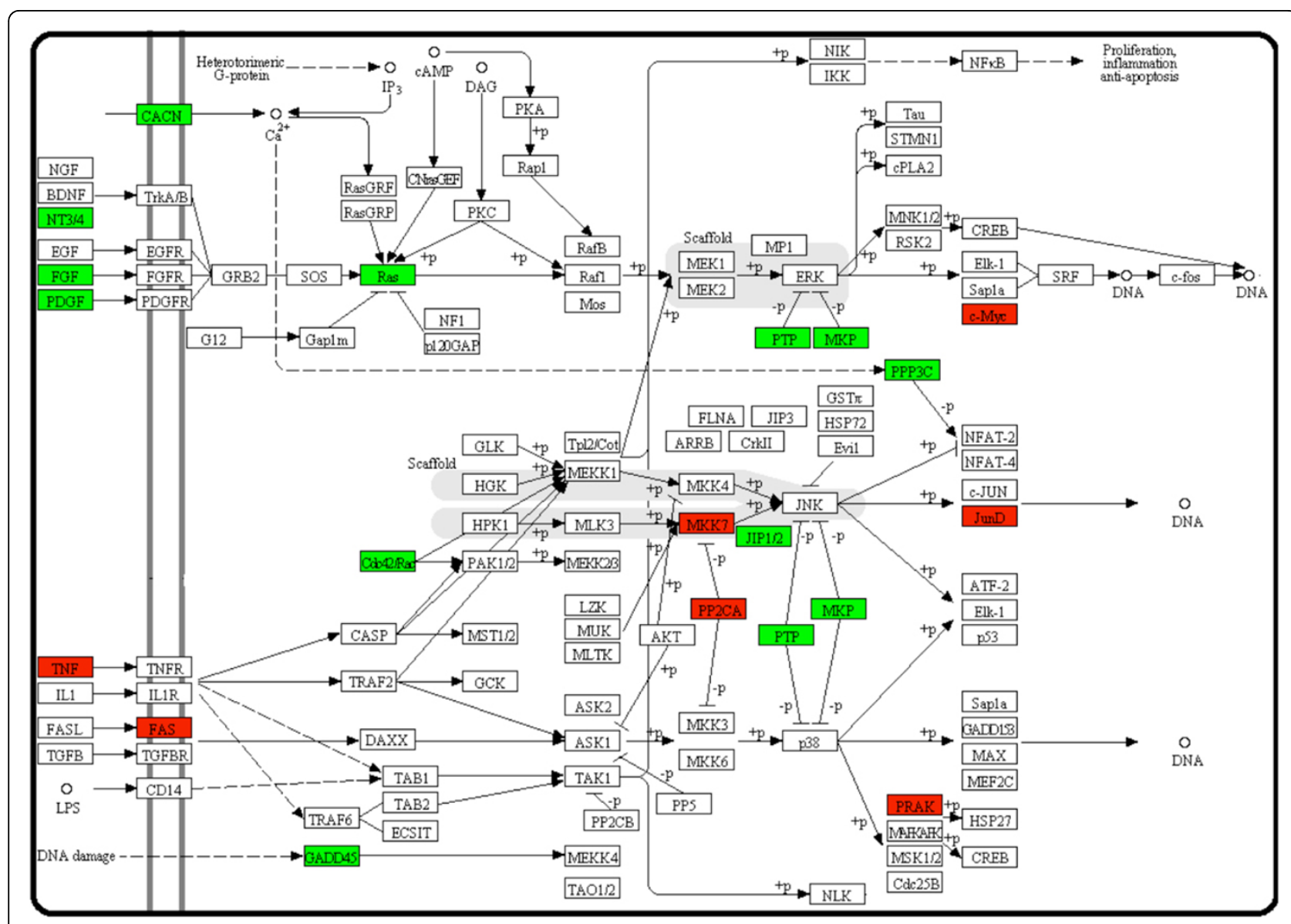

Figure 3 MAPK signalling pathway. See Figure 2 legend for the definitions of different colours. In this pathway, many important cancerrelated genes, such as Hras1, Mkk7 and Prak, were lost. 
of this pathway could lead to neoplastic proliferation which is involved in the progress of cancer cells.

Seventeen genes were found to be absent in the NMR genome (Figure 4 and Supplementary Table 4 [see additional file 1]). Among them, 4 (25\%) genes have corresponding phenotypes of cancer whereas 12 (75\%) genes were differentially expressed between cancer and normal tissues. Molecular-level studies have indicated that approximately $90 \%$ of the activating mutations of genes in this pathway could cause different cancers such as colorectal cancer [35].

Several well-studied cancer-related genes, such as Myc, Rhoa, Lef1 and Rac1, were absent in this pathway of NMR. Myc is a well-known proto-oncogene and has been frequently used to induce tumour formation in a lot of animal experiments of cancer research. Rhoa has been deeply studied and proved as a cancer-regulated gene, which controlled metastasis of tumour cells, acted as a regulator of male hormone activity in prostate cancer cells [36], and triggered a particular microvesicle signalling pathway in cancer cells [37]. Lef1 protein could interact with a number of other proteins, such as Ctbp and Nlk.
These interactions were thought to be responsible for the invasion and growth of prostate cancer [38]. Rac1 was found to be associated with DNA transcription. Previous studies have reported that activation of Rac1 mediated Twist1-induced cancer cell migration [39]. On the other hand, 12 of the NMR-missing genes in this pathway, such as Dkk4, Sox17 and Ccnd3, have not been reported to be related to any disease including cancer. It is possible that some of them are also involved in cancer formation and could be further experimentally verified.

\section{Sequence variation analysis of Class I genes}

Based on the BLASTP sequence alignment results, we found that $6349(41.2 \%)$ of the orthologs had no gaps and $12142(78.8 \%)$ orthologs only possessed a small ( $\leq 5$ amino acids) gaps, suggesting that most of the orthologous proteins between rat and NMR had rare insertions/deletions during evolution. On the other hand, 439 orthologous proteins showed significant segment insertions/deletions whose length was more than 25 amino acids. Further analysis of these inserted and deleted fragments in these proteins revealed that many

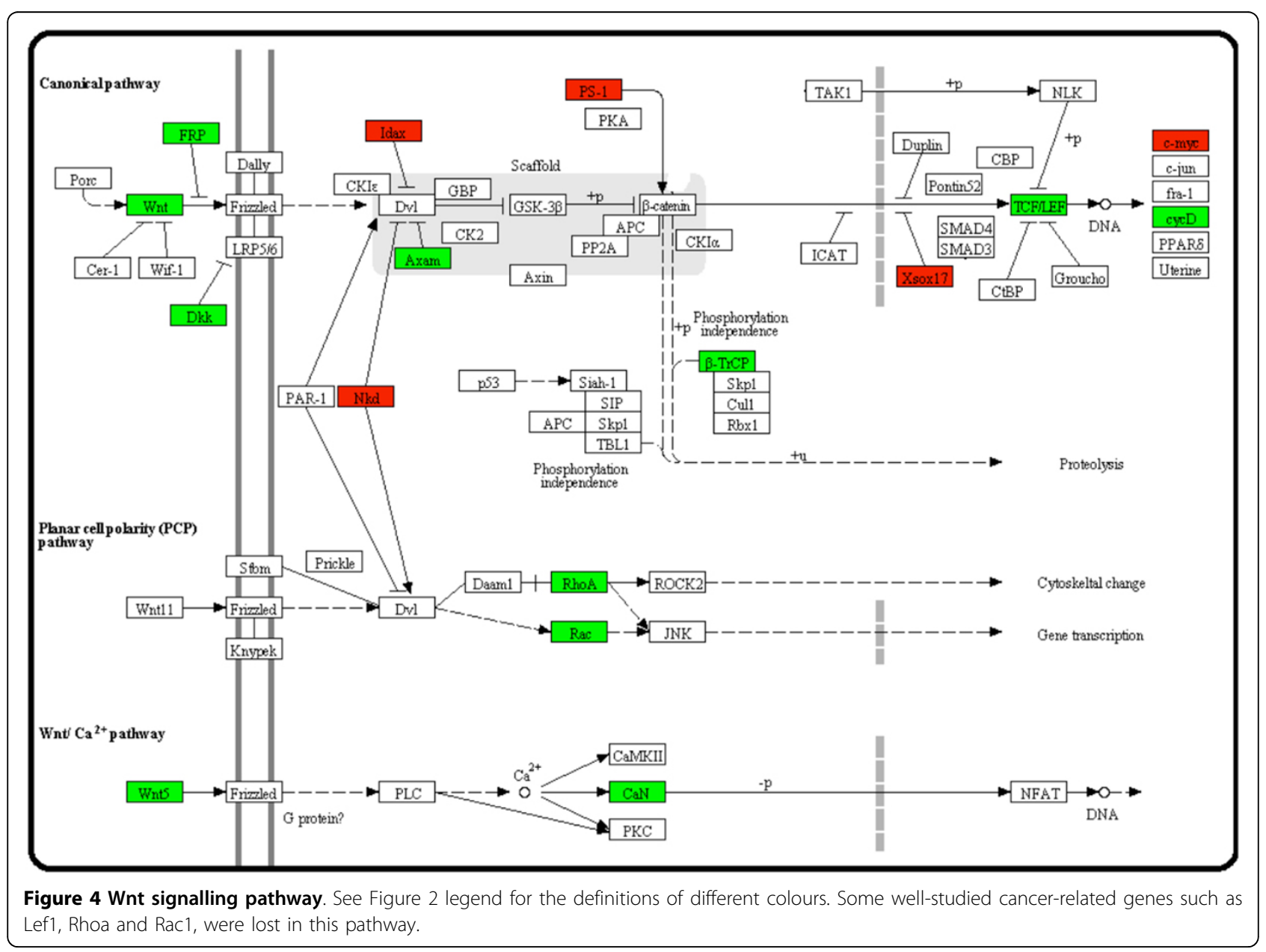


of them contained conserved sites, including functionally active sites (Supplementary Table 5 [see additional file 1]). For example, we observed that parts of the specific RNA/ DNA binding site and the specific cytokine receptor motif were deleted in the NMR Fusip1 and Nrcam proteins, respectively.

Other domains affected by the insertion/deletion of certain segments included ATP-binding, $\mathrm{Ca}^{2+}$ binding and some other metal catalytic binding sites. For example, a 30-amino-acid-long sequence fragment was found to be inserted into the putative catalytic site in NMR Ship1 when compared with its ortholog in rat. It has been previously demonstrated that the phosphate domain of Ship1 was essential for catalytic activity in vivo [40] and the loss of Ship1 could promote leukemogenesis in a virus-infected mouse model [41]. We suspect the insertion of such a long segment of Ship1 would change the function or expression of this gene. On the other hand, wrong annotation of some NMR proteins could not be excluded. Further studies are required to verify the presence of the sequence variations and their influence on the regulation or function of these proteins.

\section{Conclusions}

In this paper, a comparative genomics study was carried out to investigate the genes that were either common between rat and NMR, or specific to each of them. The majority of genes were shared by the two rodents, whereas each organism had a significant part of unique genes. Seven cancer-related protein families, such as melanoma-associated antigen family, protein kinase $\mathrm{C}$ family and HSP family were found to be significantly expanded. Further analysis of the genes absent in NMR indicated that the majority of them have been shown to be linked to many forms of cancer. Finally, some conserved functional domains were found to be possibly influenced by the insertion or deletion of certain fragments in NMR, which may change the expression or function of some of these genes. These results may provide important clues about the molecular mechanisms of cancer resistance of NMR and help identify new cancer-related genes [42] in mammals. As future topics, it is important to study such complex mechanisms from the viewpoints of network [43-46] and dynamics $[47,48]$ by further incorporating the expression data.

\section{Additional material}

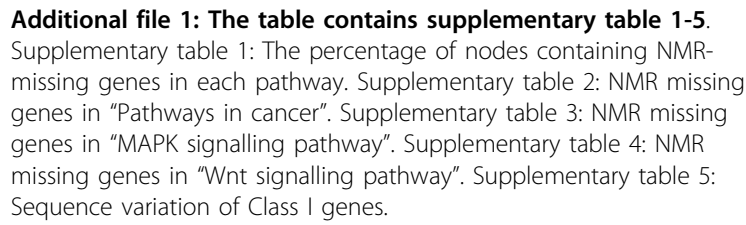

\section{List of abbreviations}

NMR: naked mole rat; KEGG: Kyoto Encyclopaedia of Genes and Genomes; NCBI: National Center for Biotechnology Information; GXA: Gene Expression Atlas; CDD: Conserved Domain Database; OMIM: Online Mendelian Inheritance in Man; PKC: protein kinase C; HSP: heat shock proteins; TERT: telomerase reverse transcriptase; GPx1: glutathione peroxidase 1; VOR: vomeronasal organ receptor

\section{Competing interests}

The authors declare that they have no competing interests.

\section{Authors' contributions}

YZ and LC designed the study. ZY carried out computational studies, including comparative genomics, sequence alignment, Pfam and KEGG analysis and wrote the manuscript. YZ and LC analyzed the data and edited the manuscript. All authors read and approved the final manuscript.

\section{Acknowledgements}

A preliminary version of this paper was published in the proceedings of IEEE ISB2012. We are also very grateful to KEGG database because of the pathway maps drawn by it.

\section{Declarations}

The publication of this article is funded by grants from the National Natural Science Foundation of China (91029301, 61134013, 31171233, 61072149), a grant from Chinese Academy of Sciences (CAS) (2012OHTP10), the Chief Scientist Program of Shanghai Institutes for Biological Sciences of CAS (2009CSP002), and the FIRST program from JSPS initiated by CSTP. This article has been published as part of BMC Systems Biology Volume 7 Supplement 2, 2013: Selected articles from The 6th International Conference of Computational Biology. The full contents of the supplement are available online at http://www.biomedcentral.com/bmcsystbiol/supplements/7/S2.

\section{Authors' details}

${ }^{1}$ Key Laboratory of Systems Biology, SIBS-Novo Nordisk Translational Research Centre for PreDiabetes, Shanghai Institutes for Biological Sciences, Chinese Academy of Sciences, Shanghai 200031, China. ${ }^{2}$ Key Laboratory of Nutrition and Metabolism, Institute for Nutritional Sciences, Shanghai Institutes for Biological Sciences, Chinese Academy of Sciences, Shanghai 200031, China. ${ }^{3}$ Collaborative Research Center for Innovative Mathematical Modelling, Institute of Industrial Science, The University of Tokyo, Tokyo 1538505, Japan.

Published: 14 October 2013

\section{References}

1. van der Horst G, Maree $L$, et al: Sperm structure and motility in the eusocial naked mole-rat, Heterocephalus glaber: a case of degenerative orthogenesis in the absence of sperm competition. BMC Evol Biol 2011, 11:351.

2. Buffenstein $R$ : The naked mole-rat: a new long-living model for human aging research. J Gerontol A Biol Sci Med Sci 2005, 60(11):1369-1377.

3. Deacon RM, Dulu TD, Patel NB: Naked mole-rats: Behavioural phenotyping and comparison with C57BL/6 mice. Behav Brain Res 2012, 20 231(1):193-200

4. Buffenstein R: Negligible senescence in the longest living rodent, the naked mole-rat: insights from a successfully aging species. J Comp Physiol B 2008, 178(4):439-45.

5. Edrey $Y H$, Hanes $M$, et al: Successful aging and sustained good health in the naked mole rat: a long-lived mammalian model for biogerontology and biomedical research. ILAR J 2011, 52(1):41-53.

6. World Health Organization: The Global Burden of Disease: 2004 Update. Geneva: World Health Organization; 2008.

7. Helmig S, Schneider J: Oncogene and tumor-suppressor gene products as serum biomarkers in occupational-derived lung cancer. Expert Rev $\mathrm{Mol}$ Diagn 2007, 7(5):555-568.

8. Woodley R, Buffenstein R: Thermogenic changes with chronic cold exposure in the naked mole-rat (Heterocephalus glaber). Comp Biochem Physiol A Mol Integr Physiol 2002, 133(3):827-834.

9. Park TJ, et al: Selective inflammatory pain insensitivity in the African naked mole-rat (Heterocephalus glaber). PLOS Biol 2008, 6(1):e13. 
10. Edrey $Y H$, Park TJ, Kang H, Biney A, Buffenstein R: Endocrine function and neurobiology of the longest-living rodent, the naked mole-rat. Exp Gerontol 2011, 46:116-123.

11. Peterson BL, Larson J, Buffenstein R, Park TJ, Fall CP: Blunted Neuronal Calcium Response to Hypoxia in Naked Mole-Rat Hippocampus. PLOS ONE 2012, 7(2):e31568, doi:10.1371/journal.pone.0031568.

12. Kim $E B$, et al: Genome sequencing reveals insights into physiology and longevity of the naked mole rat. Nature 2011, 479(7372):223-7.

13. Punta $M$, Coggill $P C$, et al: The Pfam protein families database. Nucleic Acids Res 2012, 40(Database issue):D290-301.

14. Jitao Z, Stefan W: KEGGgraph: a graph approach to KEGG PATHWAY in R and bioconductor. Bioinformatics 2009, 25(11):1470-1471.

15. Kasaikina MV, Lobanov AV, Malinouski MY, Lee BC, Seravalli J, Fomenko DE, Turanov AA, Finney L, Vogt S, Park TJ, Miller RA, Hatfield DL, Gladyshev VN: Reduced utilization of selenium by naked mole rats due to a specific defect in GPx1 expression. J Biol Chem 2011, 286(19):17005-14.

16. Griner EM, Kazanietz MG: Protein kinase $C$ and other diacylglycerol effectors in cancer. Nat Rev Cancer 2007, 7(4):281-294.

17. Ali AS, Ali $S$, et al: Exploitation of protein kinase C: a useful target for cancer therapy. Cancer Treat Rev 2009, 35(1):1-8.

18. Ricaniadis N, Kataki A, et al: Long-term prognostic significance of HSP-70, c-myc and HLA-DR expression in patients with malignant melanoma. Eur J Surg Oncol 2001, 27(1):88-93.

19. Pick E, Kluger $Y$, et al: High HSP90 expression is associated with decreased survival in breast cancer. Cancer Res 2007, 67(7):2932-2937.

20. Galluzzi L, Giordanetto F, et al: Targeting HSP70 for cancer therapy. Mol Cell 2009, 36(2):176-177.

21. Mahalingam D, Swords R, et al: Targeting HSP90 for cancer therapy. Br J Cancer 2009, 100(10):1523-1529.

22. Berx G, van Roy F: Involvement of members of the cadherin superfamily in cancer. Cold Spring Harb Perspect Biol 2009, 1(6):a003129.

23. Gama A, Schmitt F: Cadherin cell adhesion system in canine mammary cancer: a review. Vet Med Int 2012, 2012:357187.

24. Smith ES, Omerbasic D, et al: The molecular basis of acid insensitivity in the African naked mole-rat. Science 2011, 334(6062):1557-1560.

25. Van Dyke AL, Cote ML, et al: Cytokine and cytokine receptor singlenucleotide polymorphisms predict risk for non-small cell lung cancer among women. Cancer Epidemiol Biomarkers Prev 2009, 18(6):1829-1840.

26. Mirzayans $R$, Andrais $B$, et al: New Insights into p53 Signalling and Cancer Cell Response to DNA Damage: Implications for Cancer Therapy. J Biomed Biotechnol 2012, 2012:170325.

27. Hu W, Zheng RR, et al: Effects of bortezomib in sensitizing human prostate cancer cell lines to NK-mediated cytotoxicity. Asian J Androl 2012, 14(5):695-702.

28. Sastre-Perona A, Santisteban P: Role of the wnt pathway in thyroid cancer. Front Endocrinol (Lausanne) 2012, 3:31.

29. Xia J, Li Y, et al: Arsenic Trioxide Inhibits Cell Growth and Induces Apoptosis through Inactivation of Notch Signalling Pathway in Breast Cancer. Int J Mol Sci 2012, 13(8):9627-9641.

30. Liang $\mathrm{S}$, Mele J, et al: Resistance to experimental tumorigenesis in cells of a long-lived mammal, the naked mole-rat (Heterocephalus glaber). Aging Cell 2010, 9(4):626-635.

31. Martínez Q, Rodríguez G, et al: LEOPARD Syndrome: Clinical Features and Gene Mutations. Mol Syndromol 2012, 3:145-157.

32. Seluanov A, Hine C, Azpurua J, Feigenson M, Bozzella M, Mao ZY, et al: Hypersensitivity to contact inhibition provides a clue to cancer resistance of naked mole-rat. Proc Natl Acad Sci USA 2009, 106:19352-19357.

33. Zerbini LF, de Vasconcellos JF, et al: Jund-mediated repression of GADD45alpha and gamma regulates escape from cell death in prostate cancer. Cell Cycle 2011, 10(15):2583-2591.

34. Yoshizuka N, Lai M, et al: Prak suppresses oncogenic ras-induced hematopoietic cancer development by antagonizing the JNK pathway. Mol Cancer Res 2012, 10(6):810-820.

35. Giles $\mathrm{RH}$, van Es $J$, et al: Caught up in a Wnt storm: Wnt signaling in cancer. Biochim Biophys Acta 2003, 1653(1):1-24.

36. Schmidt $L$, Duncan $K$, et al: RhoA as a mediator of clinically relevant androgen action in prostate cancer cells. Mol Endocrinol 2012, 26(5):716-735.

37. Li B, Antonyak MA, et al: RhoA triggers a specific signaling pathway that generates transforming microvesicles in cancer cells. Oncogene 2012, doi: 10.1038/onc.2011.636.
38. Li YR, Wang LG, et al: LEF1 in Androgen-Independent Prostate Cancer: Regulation of Androgen Receptor Expression, Prostate Cancer Growth, and Invasion. Cancer Res 2009, 69(8):3332-3338.

39. Yang WH, Lan HY, et al: RAC1 activation mediates Twist1-induced cancer cell migration. Nat Cell Biol 2012, 14(4):366-374.

40. Conde C, Gloire G, et al: Enzymatic and non-enzymatic activities of SHIP1 in signal transduction and cancer. Biochem Pharmacol 2011, 82(10):1320-1334.

41. Lakhanpal GK, Vecchiarelli-Federico LM, Li YJ, Cui JW, Bailey ML, Spaner DE, et al: The inositol phosphatase SHIP-1 is negatively regulated by Fli-1 and its loss accelerates leukemogenesis. Blood 2010, 116:428-36.

42. Yang Z, Yan Z, Chen L: In silico identification of novel cancer-related genes by comparative genomics of naked mole rat and rat. Systems Biology (ISB), 2012 IEEE 6th International Conference 2012, 285-290, 18-20 Aug. 2012, doi: 10.1109/ISB.2012.6314151.

43. Song W, Wang J, Yang Y, Jing NH, Zhang XS, Chen L, Wu JR: Rewiring drug-activated p53-regulatory network from suppressing to promoting tumorigenesis. Journal of Molecular Cell Biology 2012, 4(4):197-206.

44. He D, Liu Z, Honda M, Kaneko S, Chen L: Coexpression network analysis in chronic hepatitis $B$ and $C$ hepatic lesion reveals distinct patterns of disease progression to hepatocellular carcinoma. Journal of Molecular Cell Biology 2012, 4(3):140-152.

45. Wen Z, Liu Z, Liu Z, Zhang Y, Chen L: An integrated approach to identify causal network modules of complex diseases with application to colorectal cancer. Journal of the American Medical Informatics Association 2012, doi:10.1136/amiajnl-2012-001168.

46. Wang J, Sun Y, Zheng S, Zhang X, Zhou H, Chen L: APG: an Active Protein-Gene Network Model to Quantify Regulatory Signals in Complex Biological Systems. Scientific Reports 2013, DOI: 10.1038/srep01097.

47. Chen L, Liu R, Liu Z, Li M, Aihara K: Detecting early-warning signals for sudden deterioration of complex diseases by dynamical network biomarkers. Scientific Reports 2012, 2:342.

48. Zhu H, Shyama Prasad Rao R, Liu J, Zeng T, Chen L: Reconstructing dynamic gene regulatory networks from sample-based transcriptional data. Nucleic Acids Research 2012, doi:10.1093/nar/gks860.

doi:10.1186/1752-0509-7-S2-S5

Cite this article as: Yang et al:: Investigation of anti-cancer mechanisms by comparative analysis of naked mole rat and rat. BMC Systems Biology 2013 7(Suppl 2):S5.

\section{Submit your next manuscript to BioMed Central and take full advantage of:}

- Convenient online submission

- Thorough peer review

- No space constraints or color figure charges

- Immediate publication on acceptance

- Inclusion in PubMed, CAS, Scopus and Google Scholar

- Research which is freely available for redistribution

Submit your manuscript at www.biomedcentral.com/submit
C Biomed Central 